\title{
A rapid detection of multidrug-resistant Mycobacterium tuberculosis by a nitrate reductase assay on blood agar
}

\author{
Ahmet Yilmaz Coban+', Yeliz Tanriverdi Cayci ${ }^{1}$, Aydin Deveci², \\ Alper Akgunes ${ }^{3}$, Meltem Uzun ${ }^{4}$, Belma Durupinar ${ }^{1}$

\begin{abstract}
'Department of Medical Microbiology ${ }^{2}$ Department of Clinical Microbiology and Infectious Disease, Medical School, Ondokuz Mayis University, Samsun, Turkey ${ }^{3}$ Department of Microbiology and Clinical Microbiology, Clinical Microbiology Laboratory, Samsun Chest Diseases Hospital, Samsun, Turkey ${ }^{4}$ Istanbul Medical School, Istanbul University, Istanbul, Turkey
\end{abstract}

The susceptibility of 49 Mycobacterium tuberculosis clinical isolates to isoniazid (INH) and rifampisin (RIF) (28 multi-drug resistant-tuberculosis samples) was determined by a nitrate reductase assay (NRA) on blood agar. Agreement between the NRA and other testing methods was found to be $93.8 \%$ for both INH and RIF. The sensitivity, specificity, positive predictive value and negative predictive value for INH were $92.8 \%, 94.2 \%, 86.6 \%$ and $97 \%$, respectively. The sensitivity, specificity, positive predictive value and negative predictive value for RIF were $90.4 \%$, $96.4 \%, 95 \%$ and $93.1 \%$. In conclusion, we show here that blood agar can be used effectively for the NRA test.

Key words: Mycobacterium tuberculosis - nitrate reductase assay - blood agar

Tuberculosis remains an important public health problem and has recently been increasing in incidence (Affolabi et al. 2007). The early detection of antimicrobial susceptibility is especially important for creating an appropriate treatment protocol for patients with tuberculosis. Multi-drug resistant (MDR) tuberculosis isolates present a major challenge for tuberculosis control programs (Shikama et al. 2009). Therefore, microbial susceptibility to at least isoniazid (INH) and rifampisin (RIF) should be ascertained to diagnose MDR tuberculosis. Conventional methods [proportion methods in Löwenstein-Jensen (LJ) and Middlebrook 7H10 agar] for tuberculosis drug susceptibility testing have many disadvantages, including being labour intensive and time consuming. Although several rapid automated testing systems (Bactec 460-TB, Bactec MGIT 960, ESP Culture System II) are available, they cannot be used effectively in developing countries due to their cost (CLSI/ NCCLS 2003, Shikama et al. 2009).

Developing alternative methods to conventional and rapid automated systems is of great importance. The resazurin microplate method (REMA), microscopic observation drug susceptibility, nitrate reductase assay (NRA), MTT test and broth microdilution method are a few examples of these alternative methods (Palomino et al. 2002, Coban et al. 2004a, b, Mengatto et al. 2006). The NRA test is typically performed on LJ medium; this presents several disadvantages, including the required cooking preparation and potential interactions between antibiotics and phospholipids in the medium (Kent \& Kubica 1985). We aimed to investigate INH and RIF

\footnotetext{
+ Corresponding author: cobanay2003@yahoo.com.tr

Received 19 November 2010

Accepted 16 February 2011
}

susceptibility for determining MDR tuberculosis using an NRA test on blood agar, a method which has recently been developed to test the antibiotic susceptibility of tuberculosis bacilli (Coban et al. 2006).

Forty-nine Mycobacterium tuberculosis clinical isolates, including $28 \mathrm{MDR}$ isolates and the reference strains H37Rv (susceptible to all drugs), ATCC 35822 (INH resistant) and ATCC 35820 (streptomycin resistant), were tested in this study. Antibacterial resistance patterns of the clinical isolates are summarised in Table I. Drug susceptibility testing of all isolates was determined by Bactec $460 \mathrm{~TB}$, which is considered the benchmark automated system and is commonly used in diagnostic laboratories. INH and RIF powders were obtained from Sigma-Aldrich, Germany. Stock solutions were prepared according to the manufacturer's instructions and stored at $-80^{\circ} \mathrm{C}$.

Blood agar-based medium (bioMérieux, SA, Marcy I'Etoile France) was prepared according to the manufacturer's recommendations with some modifications. Five millilitres of glycerol per litre of medium and potassium nitrate $(1000 \mu \mathrm{g} / \mathrm{mL})$ were added to the medium, which was then sterilised using an autoclave. After being cooled to $45^{\circ} \mathrm{C}$, the medium was supplemented with $5 \%$ defibrinated sheep blood. Media with antibiotics were prepared from stock antibiotic solutions. Antibiotic concentrations identified by CLSI/NCCLS (2003) for Middlebrook $7 \mathrm{H} 10$ agar were used in the blood agar. Five millilitres of medium with final concentrations of $0.2 \mu \mathrm{g} / \mathrm{mL}$ INH and $1 \mu \mathrm{g} / \mathrm{mL}$ RIF was prepared in screw-cap glass tubes and kept in an oblique position to solidify. Three drug-free control media were prepared for each isolate.

Two or three colonies freshly grown on LJ medium were dispensed and vortexed for $30 \mathrm{~s}$ in screw-cap tubes containing $3 \mathrm{~mL}$ of phosphate buffer and 8-10 glass beads. The tubes were then kept in a vertical position for $30 \mathrm{~min}$ at room temperature to sediment large particles and aerosols. The supernatant was then transferred to another tube and adjusted to McFarland 1 standard; in 
addition, bacterial suspension was then diluted 1:10 with phosphate buffer from the bacterial suspension and adjusted to McFarland 1 standard (Angeby et al. 2002).

Two hundred microlitres of bacterial suspension adjusted to McFarland 1 standard was inoculated into a screw-cap tube containing blood agar with antibiotic. An equal volume of the 1:10 dilution was inoculated into three screw-cap tubes containing drug-free blood agar. All tubes were incubated at $37^{\circ} \mathrm{C}$. Five hundred microlitres of freshly prepared Griess reagent (1 part 50\% hydrochloric acid, 2 parts $0.2 \%$ sulphanilamide and 2 parts $0.1 \% n$-1-naphthyl-ethylenediamine dihydrochloride) was added to the drug-free tubes on the seventh day of incubation. The Griess reagent was added to tubes containing antibiotics when a colour change was observed. Results were reported according to colour changes; if a colour change was not observed, the incubation period was extended to the 10th and 14th days (Angeby et al. 2002).

Results were obtained on the seventh day for 44 isolates and on the 10th day for five isolates. One false INHresistant isolate and two false INH-susceptible isolates were found by NRA, as determined by Bactec 460 TB. False RIF resistance and susceptibility by NRA comparing with Bactec $460 \mathrm{~TB}$ were determined in two isolates and one isolate, respectively. Agreement was found to be $93.8 \%$ for both INH and RIF. The sensitivity, specificity, positive predictive value and negative predictive value for INH were found to be $92.8 \%, 94.2 \%, 86.6 \%$ and $97 \%$, respectively. The sensitivity, specificity, positive predictive value and negative predictive value for RIF were $90.4 \%$, $96.4 \%, 95 \%$ and $93.1 \%$ (Table II), respectively.

The NRA method has been directly or indirectly compared with other reference methods, including the Bactec 460 TB, Bactec MGIT 960, REMA test and proportion method in LJ medium, for determining antibiotic susceptibility in tuberculosis (Sanchotene et al. 2008, Ani et al. 2009, Rosales et al. 2009, Shikama et al. 2009, Visalakshi et al. 2010b). Forty-nine M. tuberculosis clinical isolates were indirectly tested by NRA in this study. The specificities of the NRA test for INH and RIF were found to be $94.2 \%$ and $96.4 \%$ and the sensitivities were found to be $92.8 \%$ and $90.4 \%$, respectively. NRA results agreement was found to be $93.8 \%$ for both drugs. Results were available between days seven-14, with the majority being available by the 10th day. In this study, results were obtained on the seventh day for 44 isolates and on the 10th day for five isolates.

NRA is used to determine secondary drug susceptibility in addition to its use for determining the susceptibility of $M$. tuberculosis clinical isolates to primary anti-tuberculosis drugs. The sensitivity of NRA for kanamycin, ethionamid, ofloxacin, cycloserine and paraaminosalicylic acid susceptibility has been reported to be $86.4-100 \%$ and NRA specificity has been found to be 98.4-100\% (Visalakshi et al. 2010a). Martin et al. (2005) have shown complete agreement between the results of the NRA and the reference method for determining ofloxacin susceptibility. They also performed an NRA test in Middlebrook 7H11 instead of in LJ medium. Devasia et al. (2009) have reported that NRA tests were sensitive and specific in determining the fluoroquinolone resistance of M. tuberculosis clinical isolates. It has also been shown that an adapted NRA test could be used to determine primary anti-tuberculosis drug susceptibility (Syre et al. 2003, 2006). Affolabi et al. (2008) have evaluated NRA using a liquid medium in assaying the susceptibility of

\section{TABLE I}

Resistance profile of tested Mycobacterium tuberculosis clinical isolates

\begin{tabular}{lc}
\hline Resistance profile & Number of isolates \\
\hline INH & 6 \\
STM & 2 \\
ETM & 2 \\
INH + ETM & 1 \\
Multidrug resistant isolates & 9 \\
INH + RIF & 10 \\
INH + RIF + ETM & 9 \\
INH + RIF + STM + ETM & 10 \\
Susceptible to all drugs & 49 \\
\hline Total &
\end{tabular}

ETM: ethambutol; INH: isoniazid; RIF: rifampicin; STM: streptomycin.

TABLE II

Comparison of blood agar results and BACTEC 460-TB system results

Results of BACTEC 460 TB system

\begin{tabular}{|c|c|c|c|c|c|c|c|c|}
\hline Drugs & $\begin{array}{l}\text { Results on } \\
\text { blood agar }\end{array}$ & Resistant & Susceptible & $\begin{array}{c}\text { Sensitivity } \\
(\%)\end{array}$ & $\begin{array}{c}\text { Specificity } \\
(\%)\end{array}$ & $\begin{array}{l}\text { PPV } \\
(\%)\end{array}$ & $\begin{array}{c}\text { NPV } \\
(\%)\end{array}$ & $\begin{array}{c}\text { Agreement } \\
(\%)\end{array}$ \\
\hline \multirow[t]{2}{*}{ INH } & Resistant & 33 & 1 & 92.8 & 94.2 & 86.6 & 97 & 93.8 \\
\hline & Susceptible & 2 & 13 & - & - & - & - & - \\
\hline \multirow[t]{2}{*}{ RIF } & Resistant & 27 & 2 & 90.4 & 96.4 & 95 & 93.1 & 93.8 \\
\hline & Susceptible & 1 & 19 & - & - & - & - & - \\
\hline
\end{tabular}

INH: isoniazid; NPV: negative predictive value; PPV: positive predictive value; RIF: rifampicin. 
144 clinical isolates to INH and RIF. They found the test sensitivity to be $100 \%$ for both drugs and the specificity for INH and RIF to be $99.2 \%$ and $99.3 \%$, respectively. It has been also demonstrated that NRA is a reliable method for determining the minimal inhibitory concentration of INH, RIF and ethambutol (Kumar et al. 2005).

Blood agar has been shown to be useful in testing the drug susceptibility of tuberculosis. This study shows that it can also be used to perform an NRA test. The critical concentration $(1 \mu \mathrm{g} / \mathrm{mL})$ recommended for drug susceptibility testing on Middlebrook 7H10 for RIF was used instead of the critical concentration $(40 \mu \mathrm{g} / \mathrm{mL})$ recommended for testing on LJ medium. Unlike LJ medium, blood agar does not require cooking for solidification and does not have a risk of antibiotic interactions with any of its contents. This is in marked contrast to LJ, in which phospholipids can interact with antibiotics.

In conclusion, we have shown that blood agar can be used to perform NRA tests, which are useful in drug susceptibility testing for determining MDR tuberculosis in developed and developing countries.

\section{REFERENCES}

Affolabi D, Odoun M, Martin A, Palomino JC, Anagonou S, Portaels F 2007. Evaluation of direct detection of Mycobacterium tuberculosis rifampin resistance by a nitrate reductase assay applied to sputum samples in Cotonou, Benin. J Clin Microbiol 45: 2123-2125.

Affolabi D, Odoun M, Sanoussi N, Martin A, Palomino JC, Kestens L, Anagonou S, Portaels F 2008. Rapid and inexpensive detection of multidrug-resistant Mycobacterium tuberculosis with the nitrate reductase assay using liquid medium and direct application to sputum samples. J Clin Microbiol 46: 3243-3245.

Angeby KA, Klintz L, Hoffner SE 2002. Rapid and inexpensive drug susceptibility testing of Mycobacterium tuberculosis with a nitrate reductase assay. J Clin Microbiol 40: 553-555.

Ani AE, Daylop YB, Agbaji O, Idoko J 2009. Drug susceptibility test of Mycobacterium tuberculosis by nitrate reductase assay. $J$ Infect Dev Ctries 3: 16-19.

CLSI/NCCLS - Clinical and Laboratory Standards Institute/National Committee for Clinical Laboratory Standards 2003. Susceptibility testing of mycobacteria, nocardiae and other aerobic actinomycetes; approved standard, 2nd ed., M24-A2, CLSI, Wayne, 9 pp.

Coban AY, Birinci A, Ekinci B, Durupinar B 2004a. Drug susceptibility testing of Mycobacterium tuberculosis with nitrate reductase assay. Int J Antimicrob Agents 24: 304-306.

Coban AY, Birinci A, Ekinci B, Durupinar B 2004b. Drug susceptibility testing of Mycobacterium tuberculosis by the broth microdilution method with 7H9 broth. Mem Inst Oswaldo Cruz 99: 111-113.

Coban AY, Cihan CC, Bilgin K, Uzun M, Akgunes A, Cetinkaya E, Durupinar B 2006. Blood agar for susceptibility testing of $M y$ cobacterium tuberculosis against first-line drugs. Int $J$ Tuberc Lung Dis 10: 450-453.
Devasia RA, Blackman A, May C, Eden S, Smith T, Hooper N, Maruri F, Stratton C, Shintani A, Sterling TR 2009. Fluoroquinolone resistance in Mycobacterium tuberculosis: an assessment of MGIT 960, MODS and nitrate reductase assay and fluoroquinolone cross-resistance. J Antimicrob Chemother 63: 1173-1178.

Kent PT, Kubica GP 1985. Safety in the laboratory. In Public health mycobacteriology. A guide for the level III laboratory, Press US Department of Health and Human Services, Public Health Service, Centers for Disease Control and Prevention, Atlanta, p. 5-10.

Kumar M, Khan IA, Verma V, Kalyan N, Qazi GN 2005. Rapid, inexpensive MIC determination of Mycobacterium tuberculosis isolates by using microplate nitrate reductase assay. Diagn Microbiol Infect Dis 53: 121-124.

Martin A, Palomino JC, Portaels F 2005. Rapid detection of ofloxacin resistance in Mycobacterium tuberculosis by two low-cost colorimetric methods: resazurin and nitrate reductase assays. J Clin Microbiol 43: 1612-1616.

Mengatto L, Chiani Y, Imaz MS 2006. Evaluation of rapid alternative methods for drug susceptibility testing in clinical isolates of $M y$ cobacterium tuberculosis. Mem Inst Oswaldo Cruz 101: 535-542.

Palomino JC, Martin A, Camacho M, Guerra H, Swings J, Portaels F 2002. Resazurin microtiter assay plate: simple and inexpensive method for detection of drug resistance in Mycobacterium tuberculosis. Antimicrob Agents Chemother 46: 2720-2722.

Rosales S, Pineda-García L, Andino N, Almendarez N, Membreño H, Hoffner SE 2009. Evaluation of the nitrate reductase assay for rapid detection of extensively drug-resistant tuberculosis. Int J Tuberc Lung Dis 13: 1542-1549.

Sanchotene KO, von Groll A, Ramos D, Scholante AB, Honscha G, Valença M, Scaini CJ, da Silva PEA 2008. Comparative evaluation of the nitrate reductase assay and the resazurin microtitre assay for drug susceptibility testing of Mycobacterium tuberculosis against first line anti-tuberculosis drugs. Braz J Microbiol 39: 16-20.

Shikama ML, Ferro e Silva R, Villela G, Sato DN, Martins MC, Giampaglia CM, da Silva RF, Ferro e Silva P, da Silva Telles MA, Martin A, Palomino JC 2009. Multicentre study of nitrate reductase assay for rapid detection of rifampicin-resistant $M$. tuberculosis. Int J Tuberc Lung Dis 13: 377-380.

Syre H, Phyu S, Sandven P, Bjorvatn B, Grewal HM 2003. Rapid colorimetric method for testing susceptibility of Mycobacterium tuberculosis to isoniazid and rifampin in liquid cultures. J Clin Microbiol 41: 5173-5177.

Syre H, Valvatne H, Sandven P, Grewal HM 2006. Evaluation of the nitrate-based colorimetric method for testing the susceptibility of Mycobacterium tuberculosis to streptomycin and ethambutol in liquid cultures. J Antimicrob Chemother 57: 987-991.

Visalakshi P, Arora J, Behera D 2010a. Evaluation of nitrate reductase assay for rapid detection of resistance to second-line drugs in Mycobacterium tuberculosis in a tertiary care hospital. Diagn Microbiol Infect Dis 68: 236-240.

Visalakshi P, Meharwal SK, Myneedu VP, Behera D 2010b. Evaluation of direct method of drug susceptibility testing of Mycobacterium tuberculosis to rifampicin and isoniazid by nitrate reductase assay in a national reference laboratory. Diagn Microbiol Infect Dis 66: 148-152. 